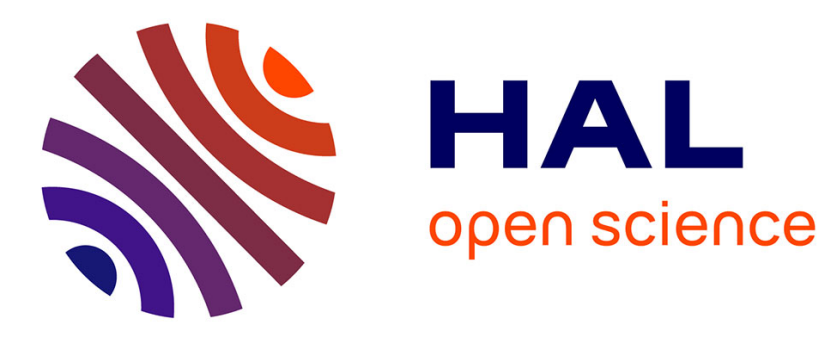

\title{
Mathematical Programming Models for Annual and Weekly Bloodmobile Collection Planning
}

\author{
Edgar Alfonso Lizarazo, Vincent Augusto, Xiaolan Xie
}

\section{To cite this version:}

Edgar Alfonso Lizarazo, Vincent Augusto, Xiaolan Xie. Mathematical Programming Models for Annual and Weekly Bloodmobile Collection Planning. IEEE Transactions on Automation Science and Engineering, 2015, 12 (1), pp.96-105. 10.1109/TASE.2014.2329571 . hal-01244989

\author{
HAL Id: hal-01244989 \\ https://hal.science/hal-01244989
}

Submitted on 11 Mar 2016

HAL is a multi-disciplinary open access archive for the deposit and dissemination of scientific research documents, whether they are published or not. The documents may come from teaching and research institutions in France or abroad, or from public or private research centers.
L'archive ouverte pluridisciplinaire HAL, est destinée au dépôt et à la diffusion de documents scientifiques de niveau recherche, publiés ou non, émanant des établissements d'enseignement et de recherche français ou étrangers, des laboratoires publics ou privés. 


\title{
Mathematical Programming Models for Annual and Weekly Bloodmobile Collection Planning
}

\author{
Edgar Alfonso, Vincent Augusto, and Xiaolan Xie, Senior Member, IEEE
}

\begin{abstract}
In this paper, we propose a two-step bloodmobile collection planning framework. The first step is the annual planning to determine weeks of collection at each mobile site in order to ensure regional self-sufficiency of blood supply. The second step is the detailed weekly planning to determine days of collections at each mobile site and to form corresponding transfusion teams. Only key resource requirements are considered for annual planning while detailed resource requirements and transportation times are considered for weekly planning. Two Mixed Integer Programming models are proposed for annual planning by assuming fixed or variable mobile collection frequencies. A new donation forecast model is proposed based on population demographics, donor generosity and donor availability. A new concept of bloodmobile collection configurations is proposed for compact and efficient mathematical modeling of weekly planning in order to minimize the total working time. Field data from the French Blood Service (EFS) in the Auvergne-Loire Region are used to design numerical experiments and to assess the efficiency of the proposed models.
\end{abstract}

Note to Practitioners-Blood collection is a crucial activity in healthcare and ensuring regional self-sufficiency of blood supply is a mission of regional blood services. Bloodmobile collection is particularly important as over $70 \%$ of blood is collected at mobile sites. Efforts are made in this paper to develop applicable mathematical models for bloodmobile collection planning by taking into account relevant information. Donation forecast is made by taking into account demographics, donor generosity and availability. Blood sufficiency is modeled by blood inventory, regional donations and blood imported from or offered to other regions. Relevant resources are considered including equipment for blood preparation and qualification, physicians, nurses, secretaries and drivers. Regulations concerning donors and staff personnel are considered. Numerical experiments based on field data show the benefits of appropriate bloodmobile collection planning.

Index Terms-Bloodmobile collection planning, regional selfsufficiency, donation forecast, transportation time

This paragraph of the first footnote will contain the date on which you submitted your paper for review. This work is supported in part by EN3S, in part by Agence Nationale de Recherche (ANR-11-TECS-010-04), in part by Natural Science Foundation of China (71131005), in part by specialized research fund for the doctoral program of higher education (20120073110040), and in part by Universidad de La Sabana, Colombia.

All authors are with the Centre for Biomedical and Healthcare Engineering, LIMOS CNRS UMR 6158-IEOR, Ecole Nationale Supérieure des Mines, Saint Etienne, France. All correspondence to: xie@emse.fr.

Edgar Alfonso is also with the Logistics Systems Group, Universidad de la Sabana, Colombia.

Xiaolan Xie is also with the Department of Industrial Engineering and Management, Shanghai Jiao Tong University, Shanghai, China.

\section{INTRODUCTION}

$\mathrm{B}$ LOOD donations are a crucial part of any healthcare system in the world. Every country has a specific organization to collect sufficient blood from safe donors, in order to meet national needs [1]. Doctors and surgeons rely on blood donations to carry out life-saving and life-enhancing treatments. In particular, non-paid blood donation through voluntary donors is recognized as crucial for the safety and sustainability of the national supply of labile blood products.

Blood transfusion services responsible for blood collection must (i) ensure an adequate, constant and stable supply of whole blood and blood products and (ii) ensure the quality and safety of the blood products for patients whose life or wellbeing depends on a blood transfusion. Since whole blood and derived blood products such as red blood cells or platelet have a limited lifespan, collection, inventory control and logistics of such products must be handled carefully in order to deliver enough blood products to health facilities (hospitals, clinics, emergency services, etc.). The timely availability of whole blood and blood products is essential for these health facilities. In this study, we focus on blood collection systems which are a crucial component of the blood supply chain.

The blood collection in France is performed by EFS (Etablissement Français du Sang or French Blood Service) in two different configurations: at fixed blood collection sites and at mobile sites. About $72 \%$ of the blood is collected at mobile sites in 2011 [2]. Over 40,000 mobile collections are organized each year. Mobile collections must be carefully planned in order to ensure the needs of health services, taking into account variability of demand, production capacity and discrepancies between regions in the country. For these reasons it is important to optimize the organization of bloodmobile collections.

Although the literature on blood supply chains is rich, the literature on blood collection systems is sparse. Most studies on blood product supply chain neglect the detailed planning and optimization of blood collection systems and the literature on blood collection systems is rare and focused on operational level. A recent study [3] on supply chain management of blood products shows that inventory management is the main focus in terms of the number of publications while collection planning is in the last place. There are nevertheless some studies related to this paper. Regional and temporal variations of weekly donations were analyzed in [4] with data from three geographically diverse American Red Cross Blood Services 
from 1995 to 2005. Mixed Integer Programming (MIP) models were proposed in [5] to address the location-allocation issues for blood services regionalization of the Turkish Red Crescent Society. One model consists in redistributing the mobile units to each service region in order to balance the service with respect to the regional populations. A strategic overview of the blood bank supply chain was proposed in [6] and main contributions to the development of operational procedures for blood bank management were analyzed. It showed that economies of scale exist in regionalization of most of the blood bank management functions. It also presented algorithms for optimal allocation of hospital blood banks and donor sites to multiple CBSs (community blood centers) of the same region. Lean manufacturing principles were used in [10] for labor efficiency improvement of bloodmobile collection. Major improvement was claimed but no formal approach was proposed to build mobile collection planning. Finally, a single period generalized network flow model was proposed in [12] to determine the optimal flow level on each link of a regional supply chain from collection to distribution by taking into account blood perishability, systemwide cost and supply risks.

This paper, an extension of a preliminary work [11], is motivated by our collaboration with the regional blood service for the Auvergne-Loire Region, France. Given the crucial mission of self-sufficiency in regional blood supply, this paper proposes a two-step bloodmobile collection framework. The first step is the annual bloodmobile collection planning in order to ensuring regional self-sufficiency in blood supply by appropriately determining weeks of the year for collections at each mobile site. The second step performs the detailed weekly planning of bloodmobile collections to determine the day of each mobile collection and to form corresponding transfusion teams. Only major resources are considered in the annual planning while detailed capacity requirements are considered in weekly planning.

Other main scientific contributions of this paper include two annual bloodmobile collection planning models with fixed and variable collection frequencies, a weekly collection model and original concepts needed to build the models. Both annual planning models ensure regional self-sufficiency. For annual planning with variable collection frequencies, we propose a probabilistic approach to predict the expected number of donations at each mobile site based on not only the collection frequency but also demographics, donor generosity and donor availability. The weekly collection planning model relies on the original concept of bloodmobile collection configurations which represent the mobile collections performed by the same transfusion team in the same day. The use of configurations instead of individual collections makes it easier to take into account transportation times which play an important role in mobile collection planning. Further, it avoids the use of a Vehicle Routing type formulation and leads to a simple mathematical model. All these models are tested on real-data to assess their efficiency.

This paper is organized as follows. French blood collection system is described in Section II. Section III provides a mathematical model for annual bloodmobile collection planning with fixed collection frequencies. Section IV extends it to annual planning with variable collection frequencies. Section V proposes a weekly bloodmobile collection model based on the new concept of collection configurations. Section VI presents the experimental results and Section VII concludes the paper.

\section{FRENCH BLOOD TRANSFUSION SYSTEM}

In France, blood collection is organized by EFS, the sole public operator authorized to satisfy the national needs in blood products [7]. The EFS is made of 17 regional EFS services (Fig. 1). Each regional EFS manages the activities of collection, preparation, qualification and distribution of blood products of the related region. The main objective of the EFS is the national self-sufficiency of blood products under optimal conditions of quality and security. Reaching this objective is difficult due to changing demand and fluctuating blood supply.

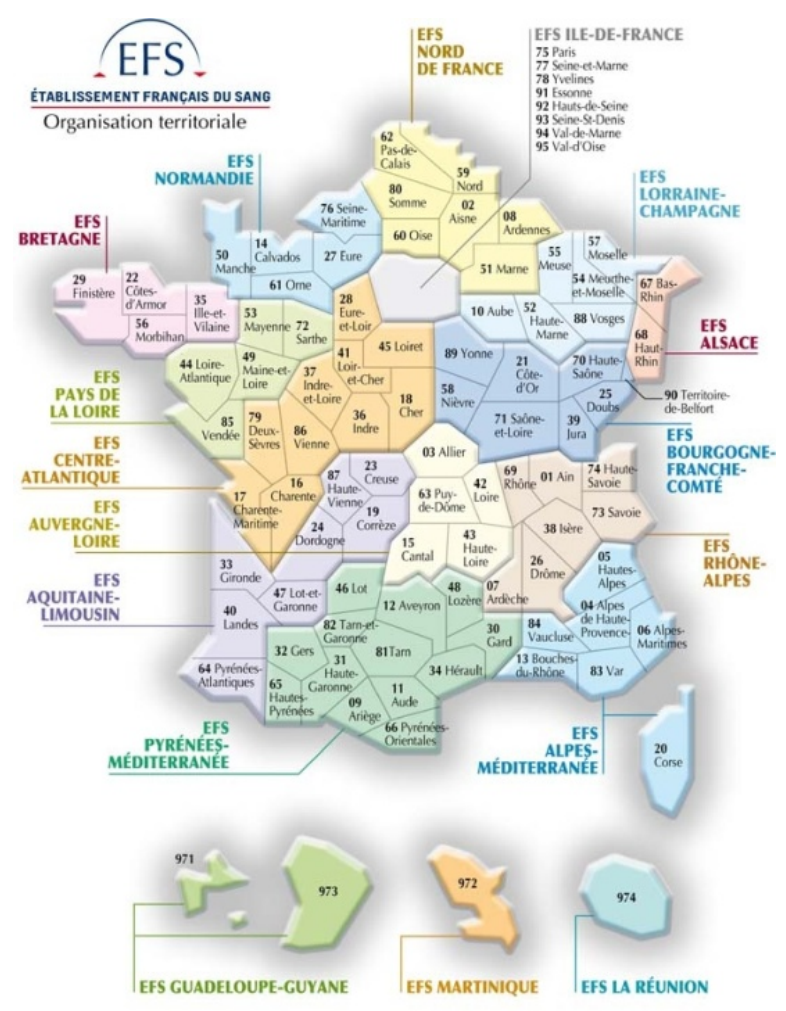

Fig. 1. Regional EFS

In order to meet the national-wide demand, the EFS has implemented a system to exchange blood productions between regional EFSs in a context of national solidarity with the aim to ensure the supply of non-self-sufficient regions. The main type of interchange between regions is related to red blood cells (RBC). The interchange of RBC to supply non-selfsufficient regions represents a significant part or the activity of the EFS.

Each regional EFS is composed of fixed sites which 
organizes the mobile collection in their corresponding territory. The percentage of donations from mobile collection is about $72 \%$ in France in 2011 [2]. In this work, we take the Auvergne-Loire Region as an example. Fig. 2 presents the organization of this regional EFS. It has 7 fixed sites but all blood components are prepared, qualified and distributed at the main site located in Saint-Etienne. The region has about 650 potential mobile collection sites.

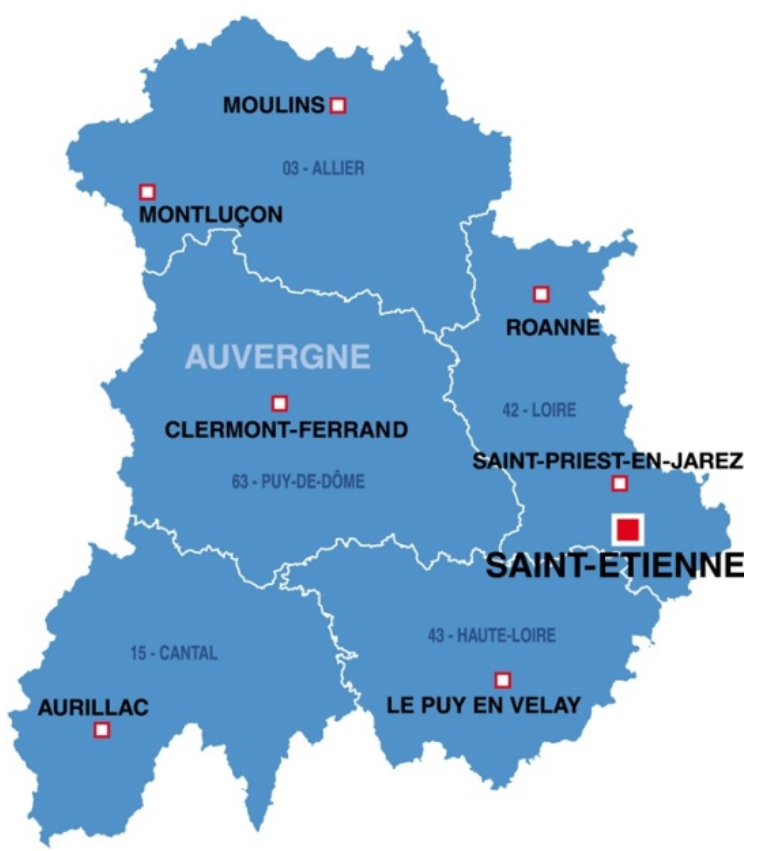

Fig. 2. EFS of the Auvergne-Loire Region

The collection of blood components at mobile sites is planned yearly based on donations of the previous years in order to match the demand of labile blood products and blood components supply. The planning must take into account constraints related to the limited human resource capacity for collection, limited capacity at the main site for blood preparation and qualification, the limited lifespan of blood products, inventory control and demands of regional health services and other regional EFS [8].

In this paper we propose mathematical models for annual and weekly bloodmobile collection planning in order to ensure the regional self-sufficiency of RBC (Red Blood Cells). Annual bloodmobile collection planning aims at ensuring the regional RBC self-sufficiency by appropriately determining the weeks of the year of collection at each mobile site under major resource constraints including availability of physicians and preparation and qualification capacity of the main fixed site. Weekly collection planning addresses the detailed planning of all mobile collections planned for a week for a fixed site, i.e. to determining the day of each collection by taking into account detailed human resource requirements including secretaries, physicians, nurses and drivers. Transportation time that is a significant part of mobile collection workload is also taken into account in weekly planning.
In the following, we present two annual planning models with fixed or variable mobile collection frequencies and one weekly planning model.

\section{ANNUAL PLANNING WITH FIXED COLLECTION FREQUENCIES}

The following assumptions are made throughout the paper:

Assumption A1: The planning horizon is one year and the time period is one week.

Assumption A2: The demand of RBC over the planning horizon is assumed known from historical records.

Assumption A3: The production capacity is given as well as the amount of human resources available at each fixed site.

Under these assumptions, the blood collection system can be described as follows. We consider a regional system composed of several fixed sites $s \in S=\{1,2, \ldots,|S|\}$ which support different mobile sites $i \in N=\{1,2, \ldots,|N|\}$ (cities, villages, firms, schools, universities, ...). Let $N_{s}$ be the set of mobile sites supported by fixed site $s$, i.e. with human resource of fixed site $s$. Each mobile site is supported by one fixed site, i.e. $N_{s} \cap N_{s^{\prime}}=\varnothing$ and $N_{1} \cup \ldots \cup N_{\bar{s}}=N$.

A planning horizon of $\bar{H}$ weeks indexed by $w \in H=\{1,2$, $\ldots,|H|\}$ is considered. The region has a weekly processing capacity of $A$ donations.

The region has a given weekly demand $D_{w}$. It can import extra blood from and export excess blood to other regions. Inventory of RBC is held but is upper bounded by $U B$ and lower bounded by SS. RBC are perishable products with a lifetime of $v=6$ weeks.

Each fixed site $s$ has a given human resource capacity $Q_{s w}$ for performing collections at either fixed and mobile sites. A resource capacity of $G_{s w}$ is needed for fixed site collections which generate $P_{s w}$ donations.

Each mobile site is also associated with (i) a set $J_{i} \subseteq H$ of weeks in which blood collection is allowed, (ii) a resource capacity requirement $b_{i}$ per collection, and (iii) a probability $R_{i}$ of donor deferral. There should be a delay of at least $K=8$ weeks between two consecutive collections at the same mobile site which ensures that no one gives blood twice within 8 weeks [9].

We make two additional assumptions in this first model.

Assumption A4: The number of mobile collections at each site over the planning horizon is fixed as in previous years.

Assumption A5: The expected number of donations of each collection at a site is known and fixed. It can be estimated based on the average number of donations at each site in the previous planning horizons.

With these assumptions, each mobile site $i$ is further associated with an expected number $F_{i}$ of donors and a given number $m_{i}$ of collections over the planning horizon.

The annual bloodmobile collection planning is represented by binary variable $x_{i w}$ equal to 1 if a collection is organized at mobile site $i$ in week $w$. Additional variables include: stock level $I_{w}$ of RBC at the beginning of week $w$, the total number $u_{w}$ of donations in week $w$, the number $u_{w}^{+}$of RBC imported 
from other regional EFSs and the number $u_{w}^{-}$of RBC offered to other regional EFSs.

The objective is to guarantee the regional self-sufficiency of $\mathrm{RBC}$, i.e. to minimize total RBC imported from other regions over the planning horizon. As a result, annual bloodmobile collection planning with fixed collection frequencies, termed model (A), can be summarized as follows:

$$
\operatorname{Min} \sum_{w \in H} u_{w}^{+}
$$

subject to:

$$
\begin{aligned}
& u_{w} \leq A, \forall w \in H \\
& \sum_{i \in N_{s}} b_{i} x_{i w}+G_{s w} \leq Q_{s w}, \forall w \in H, \forall s \in S \\
& x_{i w}=0, \forall i \in N, \forall w \notin J_{i} \\
& \sum_{s=w}^{w+K} x_{i s} \leq 1, \forall i \in N, \forall w \leq|H|-K \\
& I_{w+1}=I_{w}+u_{w}+u_{w}^{+}-u_{w}^{-}-D_{w}, \forall w \in H \\
& I_{w} \leq \sum_{p=w}^{w+v-1}\left(D_{p}+u_{p}^{-}\right), \forall w \in H \\
& S S \leq I_{w} \leq U B, \forall w \in H \\
& u_{w}=\sum_{i \in N}\left(1-R_{i}\right) F_{i} x_{i w}+\sum_{s \in S} P_{s w}, \forall w \in H \\
& \sum_{w \in J_{i}} x_{i w}=m_{i}, \forall i \in N \\
& x_{i w} \in\{0,1\}, u_{w}^{+} \geq 0, u_{w}^{-} \geq 0, \forall i \in N, \forall w \in H
\end{aligned}
$$

The objective function (1) represents the total quantity of RBC supplied by other regions, which should be minimized. Constraint (2) ensures that donations in mobile and fixed collection sites do not exceed the regional processing capacity. Constraint (3) ensures that the human resource requirements at both mobile sites and fixed sites do not exceed the resource capacity of each fixed site. Constraint (4) ensures that mobile collections are scheduled during allowed weeks. Constraint (5) ensures the minimal interval of $K$ weeks between two consecutive mobile collections at the same site. Constraint (6) is the flow balance equation. Constraint (7) forbids the distribution of perished RBC. Constraint (8) determines the suitable RBC stock level. Constraint (9) gives the total donations of the region. Constraint (10) imposes the number of mobile collections to be organized at each site in the planning horizon. Constraint (11) defines the binary variables and real variables.

\section{ANNUAL PLANNING WITH VARIABLE COLLECTION FREQUENCIES}

The assumptions A4 and A5 of fixed collection frequencies and given donations are relaxed in this section and replaced by the following assumptions.
Assumption A6: The number $m_{i}$ of collections at each mobile site $i$ is a decision variable with $m_{i} \in M$ where $M$ is the set of possible collection frequencies.

Assumption A7: The expected number of donors for each collection site depends on the collection frequency, demographics, donor generosity and donor availability.

We take into account demographics through the number of potential donors, the donor generosity through the number of donations a donor is willing to give each year, and donor availability through the probability that a donor is available when a mobile collection is organized.

Let $P_{i}$ be the number of potential donors at mobile site $i$. Let $\alpha_{i n}$ be the percentage of donors willing to donate $n$ times during the planning horizon, where $n$ is limited to 5 due to regulations about the maximum number of donations a donor can perform in one year [9]. Although this maximum number is lower for women, for the sake of simplicity, we do not distinguish male and female donations. The last parameter is the probability $q_{i}$ that a potential donor shows up to a mobile collection at site $i$.

Based on these parameters, we first determine the expected number $r_{\text {in }}$ of donations of a donor of site $i$ willing to donate $n$ times.

$$
\begin{aligned}
& r_{i 1}=\sum_{m_{1}=1}^{m}\left(1-q_{i}\right)^{m_{1}-1} q_{i} \\
& r_{i 2}=r_{i 1}+\sum_{m_{1}=1}^{m-1}\left[\left(1-q_{i}\right)^{m_{1}-1} q_{i}\left[\sum_{m_{2}=m_{1}+1}^{m}\left(1-q_{i}\right)^{m_{2}-m_{1}-1} q_{i}\right]\right] \\
& r_{i n}=r_{i n-1}+\sum_{m_{1}=1}^{m-n+1} \sum_{m_{2}=m_{1}+1}^{m_{-n+2}} \ldots \sum_{m_{n}=m_{n-1}+1}^{m}\left(1-q_{i}\right)^{m_{n}-n} q_{i}^{n}
\end{aligned}
$$

The above relations are derived as follows. $m_{n}$ indicates the collection that a donor shows up the $n$-th time. $r_{i 1}$ is the total probability that a site-i donor is available for at least one of the $m$ collections. $r_{i 2}$ is the sum of $r_{i 1}$ and the total probability that a site- $i$ donor shows up a second time. $r_{i n}$ is the sum of $r_{\text {in-1 }}$ and the total probability that a site- $i$ donor shows up an additional time.

Let $F_{\text {im }}$ be the expected number of donors present for each collection at mobile site $i$ if $m$ collections are organized at site $i$ over the horizon planning. From (13)-(15),

$$
F_{i m}=\frac{P_{i}}{m} \sum_{n=1}^{m} r_{i n} \alpha_{i n}
$$

Figure 3 is an example of the average number of donations per collection at a given mobile site. In this example, the average number of donors per collection reaches its maximum if two collections are organized and decreases as the number of collections increases. This is the result of two facts: (i) more collections reduce the loss due to donor unavailability and (ii) limited generosity of donors. 


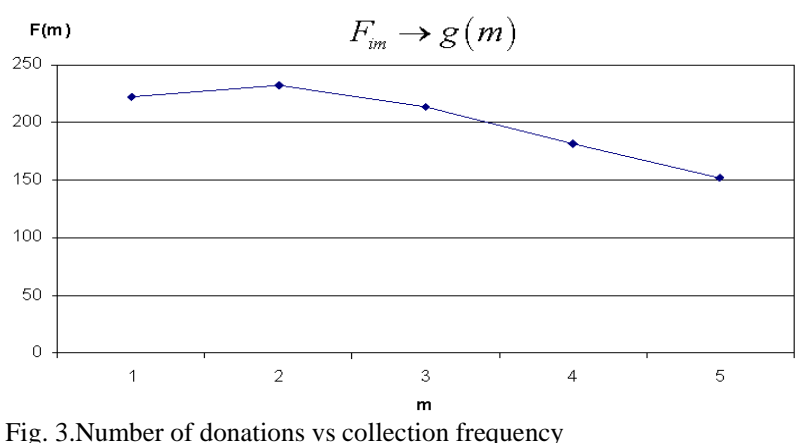

With variable mobile collection frequencies, the following decision variables are needed:

$y_{\text {im }}$ binary variable equal to 1 if $m$ mobile collections are organized at site $i$ over the planning horizon;

$S_{i w}$ non negative real variable equal to the amount of donations collected at mobile site $i$ in week $w$.

Together with the parameters $F_{\text {im }}$, annual bloodmobile collection planning with variable collection frequencies, termed model (B), can be formulated as follows:

$$
\text { Min } \sum_{w \in H} u_{w}^{+}
$$

subject to (2)-(8), (11), and

$$
\begin{aligned}
& S_{i w} \leq\left(1-R_{i}\right) \sum_{m \in M} F_{i m} y_{i m}, \forall i \in N, \forall w \in H \\
& S_{i w} \leq\left(1-R_{i}\right)\left(\max _{m \in M} F_{i m}\right) x_{i w}, \forall i \in N, \forall w \in H \\
& \sum_{w \in J_{i}} x_{i w}=\sum_{m \in M} m y_{i m}, \forall i \in N \\
& \sum_{m \in M} y_{i m}=1, \forall i \in N \\
& u_{w}=\sum_{i \in N} S_{i w}+\sum_{s \in S} P_{s w}, \forall w \in H \\
& y_{i m} \in\{0,1\}, S_{i w} \geq 0, \forall i \in N, w \in H, m \in M
\end{aligned}
$$

The objective in this model is the same as in model A. Constraints (18) and (19) ensure to allocate the value of the parameter $F_{i m}$ in the right period of time. Constraint (20) determines the equivalence between variables. Constraint (21) ensures the choice of exactly one collection frequency $m$ for each site. Constraint (22) determines the total regional collections. Constraint (23) defines the natures of the new variables.

In the above formulation, the human resource capacity requirement $b_{i}$ is assumed to be independent of the collection frequency $m_{i}$ and the expected number of donors $F_{i m}$. This is a reasonable assumption as the total time needed for a mobile collection includes large setup time needed for transportation and equipment installation. EFSs estimate the human resource requirements of a mobile collection in terms of the number of physicians and half-days needed. Some physicians perform two half-day collections in a day while others perform a single one-day collection. The change of collection frequencies over the planning horizon is unlike to turn a half-day collection into a one-day collection and to change the number of physicians needed. As a result, the human resource capacity requirement $b_{i}$ is assumed constant.

For these reasons, time needed for a mobile collection is represented in this paper in standard time units of half days and it is equal to a half-day for small collections and 2 halfdays for large collections. Combining this time with the number of physicians gives the resource requirement $b_{i}$. For example, $b_{i}=1$ for a small collection of half-day needing one physician, while $b_{i}=6$ for a large collection of one day with 3 physicians.

\section{WEEKLY BLOODMOBILE COLLECTION PLANNING}

Weekly bloodmobile collection planning starts with the results of the annual planning, i.e. the set of mobile collections planned for a given week in a territory supported by the same fixed site. It determines the day of each collection and assigns human resources to different collections. Important constraints include: (i) detailed human resource capacity for both mobile and fixed site collections, (ii) transportation time, (iii) working time restrictions of EFS personals.

\section{A. Configurations of mobile collections}

Given that an EFS transfusion team can perform more than one mobile collection, a straightforward approach is to develop a VRP-type formulation (Vehicle Routing Problems). Unfortunately this leads to highly combinatorial models that are difficult to solve.

In this paper, we use instead the concept of configurations. A configuration is the set of mobile collections that a transfusion team performs during a day. Given the large setup time and transportation time, each transfusion team can perform at most two mobile collections a day. As a result, a configuration is either a single mobile collection or two halfday collections. This is consistent with the practice of EFS.Two mobile collections $i$ and $j$ can be combined into a single configuration and performed by the transfusion team in the same day if the total time to perform the two configurations and return to EFS does not exceed the maximal daily working time $D_{\max }$. More precisely

$$
\tau_{0 i}+p_{i}+\tau_{i j}+p_{j}+\tau_{j 0}+\Delta \leq D_{\max }
$$

where $p_{i}$ and $p_{j}$ are times needed for collections $i$ and $j$ including setup times, $\tau_{i j}$ is the transportation time from location $i$ to location $j$ with location 0 denotes the fixed site, $\Delta$ denotes the total time dedicated to lunch and administrative duties.

More specifically, the set of configurations for each week 
include (i) the set of mobile collections of the week according to the annual plan, and (ii) all pairs of these collections fulfilling condition (24).

For each configuration $c$, the following parameters are defined: the number $b_{c k}$ of type- $k$ resources needed, the collection time $p_{c}$, and the transportation time $q_{c}$. If $c$ combines two collections $i$ and $j, b_{c k}$ is determined by the highest resource requirement of both collections, $p_{c}=p_{i}+p_{j}$ and $q_{c}=\tau_{0 i}+\tau_{i j}+\tau_{j 0}$.

\section{B. Parameters}

The weekly bloodmobile collection planning can be characterized by the following notation.

$T$ set of days indexed by $t$ with $T=\{1,2, \ldots, 6\}$ with $t=1$ for Monday and $t=6$ for Saturday;

$K$ set of human resource types indexed by $k$ with $K=\{\mathrm{Sc}$, $\mathrm{Ph}, \mathrm{Ns}, \mathrm{Dv}\}$ including secretaries "Sc", physicians "Ph", nurses "Ns" and drivers "Dv;

$R \quad$ set of resources indexed by $r$ with $R=\bigcup_{k \in K} R_{k}$ where $R_{k}$ denotes the set of type- $k$ resources;

$N$ set of bloodmobile collections of the week indexed by $i$;

$C$ set of bloodmobile collection configurations indexed by c with $C=\bigcup_{i \in N} C_{i}$ where $C_{i}$ denotes the set of configurations including collection $i$;

$f \quad$ daily fixed site collection;

$b_{c k}$ number of type- $k$ resources needed for configuration $c$;

$p_{c}$ total setup and collection time of configuration $c$;

$q_{c}$ total transportation time of configuration $c$;

$p_{f t}$ total collection time of a resource assigned to fixed site for day $t$;

$\Delta \quad$ lunch time and administrative duties per day per person; $D_{\max }$ maximal daily working time per person;

$W_{\max }$ maximal weekly working time per person;

$W_{\min }$ minimal weekly working time per person.

$D_{\max }, W_{\max }$ and $W_{\min }$ are from the EFS collective agreement on working times. Additionally, it is compulsory to ensure that no one works more than 5 days a week. Some lengthy organizational times such as meetings or training are not explicitly taken into account our weekly planning model but can be easily included by introducing day-dependent capacity of each resource.

\section{Weekly planning model}

Decision variables include:

$y_{c t}$ binay variable equal to 1 if configuration $c$ is planned for day $t$;

$x_{r c t}$ binary variable equal to 1 if resource $r$ is assigned to configuration $c$ in day $t$;

$x_{r f t}$ binary variable equal to 1 if resource $r$ is assigned to the fixed site collection in day $t$;

The weekly collection planning consists in planning mobile collections over the week and assigning human resources in order to minimize the total working time while realizing all mobile and fixed site collections under various working time restrictions. More precisely,

$$
\operatorname{Min} \sum_{r \in R} \sum_{t \in T}\left(\sum_{c \in C}\left(p_{c}+q_{c}+\Delta\right) x_{r c t}+\left(p_{f t}+\Delta\right) x_{r f t}\right)
$$

subject to

$\sum_{r \in R_{k}} x_{r c t} \geq b_{c k} y_{c t}, \forall t \in T, c \in C, k \in K$

$\sum_{r \in R_{k}} x_{r f t} \geq b_{f k t}, \forall t \in T, k \in K$

$\sum_{c \in C_{i}} \sum_{t \in T} y_{c t}=1, \forall i \in N$

$\sum_{c \in C} x_{r c t}+x_{r f t} \leq 1, \forall t \in T, r \in R$

$\sum_{c \in C}\left(p_{c}+q_{c}+\Delta\right) x_{r c t}+\left(p_{f t}+\Delta\right) x_{r f t} \leq D_{\max }, \forall t \in T, r \in R$

$\sum_{t \in T}\left(\sum_{c \in C}\left(p_{c}+q_{c}+\Delta\right) x_{r c t}+\left(p_{f t}+\Delta\right) x_{r f t}\right) \leq W_{\max }, \forall r \in R$

$\sum_{t \in T}\left(\sum_{c \in C}\left(p_{c}+q_{c}+\Delta\right) x_{r c t}+\left(p_{f t}+\Delta\right) x_{r f t}\right) \geq W_{\min }, \forall r \in R$

$\sum_{t \in T}\left(\sum_{c \in C} x_{r c t}+x_{r f t}\right) \leq 5, \forall r \in R$

$\sum_{c \in C} y_{c t} \geq 1, \forall t \in T, t \neq 6$

$y_{c t}, x_{r c t} \in\{0,1\}, \forall r \in R, c \in C, t \in T$

In the above model, constraint (26) ensures the resource requirement of mobile collection configurations while (27) that of fixed site collection. Constraint (28) ensures the realization of all mobile collections. Constraint (29) ensures that every resource is assigned to at most one task in a day. Constraints (30)-(32) are working time constraints. Constraint (33) ensures that no one works more than 5 days. Constraint (34) ensures at least one mobile collection during the week, a requirement of EFS.

\section{NUMERICAL RESULTS}

A. Data

Data for this study were derived from the information systems of EFS-Auvergne-Loire by taking into account the protocols about confidentiality of donor information. In total 1384 whole-blood mobile collections in 642 sites were analyzed. Statistical analysis allowed identification of the parameters for the models.

The models are tested for the "Auvergne-Loire" region with 7 fixed sites that served a total of 642 mobile collection sites. The annual planning horizon contains 52 weeks. Mobile collections are not possible in schools and universities for weeks 8-9, 16-17, 27-35, 44-45, and 51-52. For enterprises, mobile collections are not possible in weeks 31-35; 51-52. 
Mathematical models are solved using a standard MILP solver (IBM ILOG CPLEX). Annual planning models are solved on a computer with 8 parallel processors Intel Xeon 2.27 GHz and 3.23 GB of RAM memory. The computational time is $11.8 \mathrm{CPU}$ seconds for annual planning model A and 18960 seconds for annual planning model B. Weekly planning model is solved on a processor Intel Core 2 Duo $2.20 \mathrm{GHz}$ and 3.87 GB of RAM memory and CPU time ranges from 8 to 1239 seconds.

\section{B. Donation forecasting model validation}

This subsection evaluates the donation forecasting model of Section IV. Table I concerns the five most important collections with data of 2010 first and those of 2009 next. Table I gives the number of donors partitioned according to their number of donations and the annual number of donations. Data of the year with more donors are used to derive forecast of another year. For example, for site 1 , the donor population is set to 723 partitioned into five classes (357, 179, 107, 51, 29) donating 1 to 5 times, the no show probability is set to $1-p_{i}$ with $p_{i}=613 / 723$, i.e. the ratio between the annual donor numbers of 2009 and 2010. The annual donation forecast is 1349 with a forecast error of $17 \%$ for 2009.

Given the significant variation of blood donations, the forecast errors of Table I are reasonable. The no show probability has significant impact on the forecast precision. Detailed results for each site have been discussed with the EFS and validated and used for annual bloodmobile planning.

TABLE I

DONATION FORECASTING

\begin{tabular}{|c|c|c|c|c|c|}
\hline Site & Donor mix & $\begin{array}{c}\text { Annual } \\
\text { donations }\end{array}$ & No show & Forecast & Err \\
\hline 1 & $723(357,179,107,51,29)$ & 1385 & & & \\
\hline & $613(295,161,101,45,11)$ & 1155 & 0,15 & 1349 & $17 \%$ \\
\hline 2 & $813(474,199,90,37,13)$ & 1355 & 0,04 & 1392 & $3 \%$ \\
\hline & $845(486,220,95,37,7)$ & 1394 & & & \\
\hline 3 & $685(406,163,80,23,13)$ & 1129 & 0,22 & 1391 & $23 \%$ \\
\hline & $881(534,200,106,31,10)$ & 1426 & & & \\
\hline 4 & $660(331,185,88,56,-)$ & 1189 & 0,08 & 1237 & $4 \%$ \\
\hline & $718(390,179,104,45,-)$ & 1240 & & & \\
\hline 5 & $599(379,115,57,33,15)$ & 987 & 0,06 & 1047 & $6 \%$ \\
\hline & $634(368,162,70,20,14)$ & 1052 & & & \\
\hline
\end{tabular}

\section{Annual bloodmobile planning}

The results of the two annual planning models are shown in three figures. Fig. 4 compares the weekly quantity of wholeblood collected in mobile sites (red line for Model A, green line for model B). Fig. 5 compares the weekly demand of RBC and the total weekly quantities of whole-blood collected at all mobile and fixed sites. Fig. 6 shows the inventory of wholeblood over the horizon planning. The safety stock $S S$ has been fixed at 3100 units of RBC and the upper bound $U B$ at 4214 units. The initial stock is equal to $S S$.

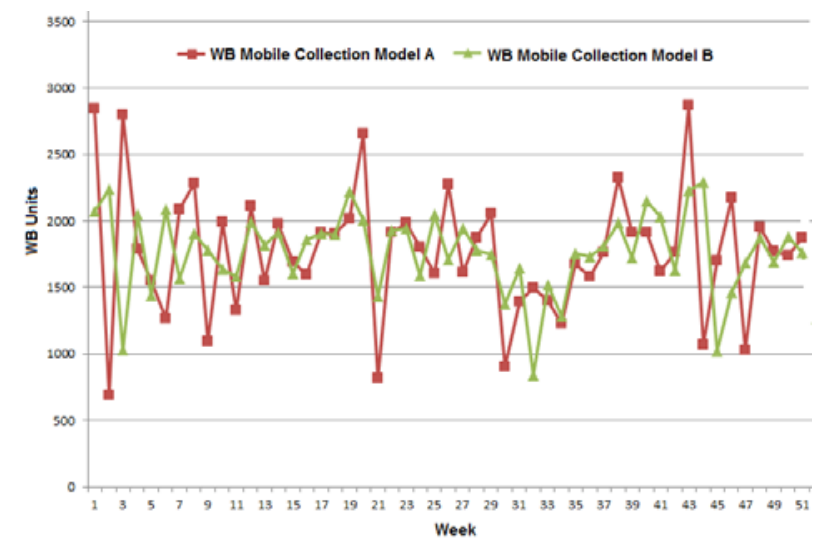

Fig. 4.Weekly whole-blood donations at mobile sites

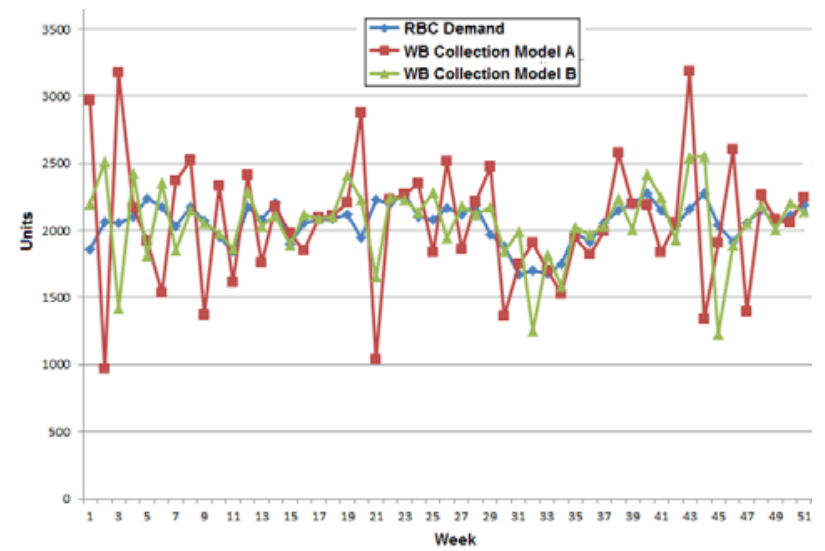

Fig. 5. Weekly whole-blood donations at all fixed and mobile sites

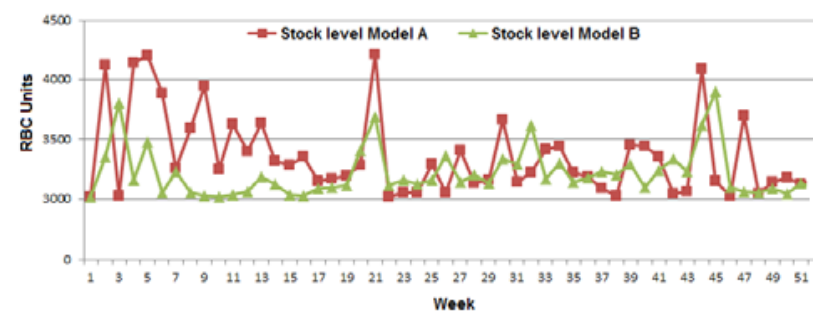

Fig. 6. Stock level of whole-blood

Both annual planning models give an optimal solution of 0 units imported from external regions, which means that the objective of self-sufficiency is reached. However, more whole-blood units are needed in model A (93763 units) than in model B (91385 units).

Model B also allows smoothed blood supply with better matching the blood collection and the demand. Model B leads to lower variability of both mobile blood collection and total blood collection. This allows model B to ensure regional selfsufficiency with lower whole-blood inventory (See figure 6).

The above results show that relaxing the assumption of fixed bloodmobile collection frequencies can indeed significantly improve the performance of blood collection with better smoothed collection activities and lower blood 
inventory.

Concerning computation times, model A runs much faster than model B. Due to larger number of variables and constraints, the memory requirement and CPU time are significantly higher with model $B$ than with model A (18,960.8 seconds on average versus 11.8 seconds). Efficient algorithms for solving these annual planning models are an issue of future research. However, since we are dealing with an annual planning, such computation time is still reasonable.

\section{Weekly bloodmobile planning}

This subsection presents weekly planning of fixed sites 1 and 2 and for four busiest weeks. Fixed site 1 has 7 physicians, 15 nurses, 5 secretaries and 4 drivers. Fixed site 2 has 5 physicians, 10 nurses, 4 secretaries and 3 drivers. Each person has an additional workload $\Delta$ of 60 minutes per working day. The collection capacity of EFS is defined by the maximal daily working time $D_{\max }$ of 600 minutes, minimal $W_{\min }$ and maximal $W_{\max }$ weekly working times of respectively 2100 and 2600 minutes.

Tables II and III give different bloodmobile collection configurations of each day, daily total workload among all staff personnel, total weekly workload, total number of mobile collections and CPU time. In these tables, configuration C195514 (129) indicates a configuration of two bloodmobile collections \#195 and \#514 with a total collection workload of 129 hours that does not include the administrative duty related load $\Delta$. Table IV shows the detailed planning of staff personnel of fixed site 1 for the week 12. The 31 human resources are numbered as follows: $1-5$ for secretaries, 6-12 for physicians, 13-27 for nurses, and 28-31 for drivers.

From Tables II and III, except for Saturday, which is dedicated to fixed site collection, the bloodmobile collections are well distributed over the week and the workload of staff personnel is well balanced.

The weekly planning model can be solved to optimality in reasonable computation time. Most cases are solved in less than 1 minute and the most difficult case (week 26 of fixed site 2) takes about 20 minutes, which is still reasonable for a weekly planning.
TABLE II

WEEKLY BLOODMOBILE PLANNING OF FIXED SITE 1

\begin{tabular}{|c|c|c|c|c|}
\hline & WK12 & WK19 & WK49 & WK23 \\
\hline Mon & $\begin{array}{l}\text { C11 (48) } \\
\text { C195-514 (129) } \\
\text { Load: 233h }\end{array}$ & $\begin{array}{l}\text { C445-632 (153) } \\
\text { Load: 233h }\end{array}$ & $\begin{array}{l}\text { C477 (46) } \\
\text { C508 (81) } \\
\text { Load: 183h } \\
\end{array}$ & \begin{tabular}{|l} 
C11 (48) \\
C515 (90) \\
Load: 194h \\
\end{tabular} \\
\hline Tues & $\begin{array}{l}\text { C103 (106) } \\
\text { C247 (40) } \\
\text { C639 (46) } \\
\text { Load: } 232 \mathrm{~h} \\
\end{array}$ & $\begin{array}{l}\text { C198 (39) } \\
\text { C303 (43) } \\
\text { C327 (41) } \\
\text { Load: } 164 h \\
\end{array}$ & $\begin{array}{l}\text { C103 (106) } \\
\text { C256 (40) } \\
\text { C258-563 (60) } \\
\text { Load:246h } \\
\end{array}$ & $\begin{array}{l}\text { C26 (91) } \\
\text { C14-190 (65) } \\
\text { Load:204h }\end{array}$ \\
\hline Weds & $\begin{array}{l}\text { C32 (83) } \\
\text { C273-371 (69) } \\
\text { Load: 200h }\end{array}$ & $\begin{array}{l}\text { C453 (115) } \\
\text { C424-481 (70) } \\
\text { Load:233h }\end{array}$ & $\begin{array}{l}\text { C16-632 (165) } \\
\text { Load 245h }\end{array}$ & \begin{tabular}{|l} 
C539 (104) \\
C144-566 (68) \\
Load:219h \\
\end{tabular} \\
\hline Thurs & $\begin{array}{l}\text { C437 (84) } \\
\text { C70-313 (68) } \\
\text { C81-317 (57) } \\
\text { Load: } 249 \mathrm{~h} \\
\end{array}$ & $\begin{array}{l}\text { C540 (98) } \\
\text { C259-500 (69) } \\
\text { Load:207h } \\
\end{array}$ & $\begin{array}{l}\text { C84 (50) } \\
\text { C319 (39) } \\
\text { C392 (44) } \\
\text { C466 (54) } \\
\text { Load: 228h } \\
\end{array}$ & \begin{tabular}{|l} 
C194 (138) \\
C546 (74) \\
Load: $252 \mathrm{~h}$ \\
\end{tabular} \\
\hline Fri & $\begin{array}{l}\text { C194 (100) } \\
\text { C220 (46) } \\
\text { C465 (42) } \\
\text { Load: } 228 \mathrm{~h}\end{array}$ & $\begin{array}{l}\text { C251 (48) } \\
\text { C582-635 (139) } \\
\text { Load: } 227 \mathrm{~h}\end{array}$ & $\begin{array}{l}\text { C418 (101) } \\
\text { C560 (59) } \\
\text { Load: } 200 \mathrm{~h}\end{array}$ & $\begin{array}{l}\text { C425 (88) } \\
\text { C513 (74) } \\
\text { Load: } 202 \mathrm{~h}\end{array}$ \\
\hline Sat & Load: 32h & Load: $68 \mathrm{~h}$ & Load: $28 \mathrm{~h}$ & Load: $48 \mathrm{~h}$ \\
\hline $\begin{array}{l}\text { WK } \\
\text { load } \\
\end{array}$ & $1173 \mathrm{~h}$ & $1131 \mathrm{~h}$ & $1131 \mathrm{~h}$ & $1118 \mathrm{~h}$ \\
\hline \# coll. & 17 & 14 & 14 & 12 \\
\hline CPU & $15 \mathrm{~s}$ & $31 \mathrm{~s}$ & $163 \mathrm{~s}$ & $8 \mathrm{~s}$ \\
\hline
\end{tabular}

TABLE III

WEEKLY BLOODMOBILE PLANNING OF FIXED SITE 2

\begin{tabular}{|c|c|c|c|c|}
\hline & WK26 & WK13 & WK22 & WK20 \\
\hline Mon & \begin{tabular}{|l} 
C85-388 (60) \\
C450-594 (78) \\
Load: 194h \\
\end{tabular} & \begin{tabular}{|l} 
C245 (38) \\
C242-315 (68) \\
Load: 170h \\
\end{tabular} & $\begin{array}{l}\text { C352 (72) } \\
\text { Load: 127h } \\
\end{array}$ & \begin{tabular}{|l} 
C25-291 (64) \\
C133-244 (54) \\
Load: 181h \\
\end{tabular} \\
\hline Tues & \begin{tabular}{|l} 
C174-297 (69) \\
C444-487 (67) \\
Load: 176h \\
\end{tabular} & $\begin{array}{l}\text { C397-611 (65) } \\
\text { C419-456 (59) } \\
\text { Load: } 164 \mathrm{~h} \\
\end{array}$ & \begin{tabular}{|l} 
C552 (55) \\
C206-463 (66) \\
Load:161h \\
\end{tabular} & \begin{tabular}{|l} 
C199 (41) \\
C87-628 (62) \\
Load:151h \\
\end{tabular} \\
\hline Weds & $\begin{array}{l}\text { C146 (50) } \\
\text { C110-120 (47) } \\
\text { Load: } 145 \mathrm{~h}\end{array}$ & $\begin{array}{l}\text { C537 (59) } \\
\text { C30-137 (50) } \\
\text { Load:157h }\end{array}$ & $\begin{array}{l}\text { C260-617 (66) } \\
\text { C365-375 (59) } \\
\text { Load 181h }\end{array}$ & $\begin{array}{l}\text { C139 (75) } \\
\text { Load:123h }\end{array}$ \\
\hline Thurs & $\begin{array}{l}\text { C146 (35) } \\
\text { C110-120 (68) } \\
\text { Load: } 143 \mathrm{~h}\end{array}$ & $\begin{array}{l}\text { C34-146 (69) } \\
\text { C391-585 (76) } \\
\text { Load:185h }\end{array}$ & $\begin{array}{l}\text { C537 (67) } \\
\text { C132-377 (69) } \\
\text { Load: } 176 \mathrm{~h}\end{array}$ & $\begin{array}{l}\text { C1-111 (55) } \\
\text { C107-451 (58) } \\
\text { Load: } 154 \mathrm{~h}\end{array}$ \\
\hline Fri & \begin{tabular}{|l} 
C47-264 (64) \\
C55-59 (61) \\
Load: 165h \\
\end{tabular} & $\begin{array}{l}\text { C594-601 (69) } \\
\text { Load: } 117 \mathrm{~h} \\
\end{array}$ & $\begin{array}{l}\text { C242-595 (67) } \\
\text { C338-576 (67) } \\
\text { Load: 174h } \\
\end{array}$ & \begin{tabular}{|l} 
C18 (68) \\
C283-484 (65) \\
Load: 172h \\
\end{tabular} \\
\hline Sat & Load: 32h & Load: 32h & Load: 36h & Load: $36 \mathrm{~h}$ \\
\hline $\begin{array}{l}\text { WK } \\
\text { load }\end{array}$ & $856 \mathrm{~h}$ & $826 \mathrm{~h}$ & $856 \mathrm{~h}$ & $819 \mathrm{~h}$ \\
\hline \# coll. & 18 & 16 & 15 & 15 \\
\hline CPU & 1239s & 11s & $54 \mathrm{~s}$ & $41 \mathrm{~s}$ \\
\hline
\end{tabular}


TABLE IV

FIXED SITE 1 PERSONNEL PLANNING FOR WEEK 12

\begin{tabular}{|c|c|c|c|c|c|}
\hline Day & Collections & Sc & $\mathrm{Ph}$ & Ns & Dv \\
\hline \multirow[t]{4}{*}{ Mon } & C11 & 5 & 8,12 & $21,25,26$ & 31 \\
\hline & C195-514 & 3,4 & $6,7,11$ & $15,17,20,23,24,27$ & 28,29 \\
\hline & Fixed site & 2 & 9,10 & $13,16,18,19$ & \\
\hline & Load & $35 \mathrm{~h}$ & $59 \mathrm{~h}$ & $112 \mathrm{~h}$ & $27 \mathrm{~h}$ \\
\hline \multirow[t]{5}{*}{ Tues } & C103 & 1,4 & $6,9,12$ & $14,15,16,19,21,24$ & 28,3 \\
\hline & C247 & 5 & 11 & $13,18,20$ & 29 \\
\hline & C639 & 3 & 7,1 & $17,26,27$ & 31 \\
\hline & Fixed site & 2 & 8 & $22,23,25$ & \\
\hline & Load & $38 \mathrm{~h}$ & $52 \mathrm{~h}$ & $113 \mathrm{~h}$ & $30 \mathrm{~h}$ \\
\hline \multirow[t]{4}{*}{ Weds } & C32 & 1,5 & $6,7,12$ & $13,14,19,23,26,27$ & 29,31 \\
\hline & C273-371 & 3 & 10,11 & $20,22,25$ & 30 \\
\hline & Fixed site & 2 & 8,9 & $16,17,18$ & \\
\hline & Load & $31 \mathrm{~h}$ & $55 \mathrm{~h}$ & $92 \mathrm{~h}$ & $23 \mathrm{~h}$ \\
\hline \multirow[t]{5}{*}{ Thurs } & C437 & 2,3 & $6,7,9$ & $13,16,18,19,23,27$ & 30,29 \\
\hline & C70-313 & 1 & 11,12 & $14,21,22$ & 31 \\
\hline & C81-317 & 4 & 10 & $20,25,26$ & 28 \\
\hline & Fixed site & 5 & 8 & $15,17,24$ & \\
\hline & Load & $40 \mathrm{~h}$ & $56 \mathrm{~h}$ & $120 \mathrm{~h}$ & $32 \mathrm{~h}$ \\
\hline \multirow[t]{5}{*}{ Fri } & C194 & 2,4 & $10,11,12$ & $15,20,22,25,26,27$ & 30,31 \\
\hline & C220 & 3 & 6,8 & $21,23,24$ & 29 \\
\hline & C465 & 1 & 9 & $16,17,18$ & 28 \\
\hline & Fixed site & 5 & 7 & $13,14,19$ & \\
\hline & Load & $37 \mathrm{~h}$ & $51 \mathrm{~h}$ & $111 \mathrm{~h}$ & $29 \mathrm{~h}$ \\
\hline
\end{tabular}

\section{CONCLUSION}

In this paper, a two-phase approach has been proposed to compute a bloodmobile collection plan at both annual tactical and weekly operational levels. The main objective is to ensure the self-sufficiency of regional blood supply. For the annual tactical planning, two models with fixed or variable collection frequencies have been proposed to compute a list of collections to perform in each week of the year. An original probabilistic model has been proposed to forecast the donations according to the collection frequency, donor demographics, generosity and availability, crucial parameters identified by EFS practitioners. At the weekly operational level, a mathematical model has been proposed to compute the collections of each day by using an original concept of bloodmobile collection configurations.

Numerical experiments have established the following numerical facts. 1) Allowing variable collection frequencies (model B) ensures self-sufficiency of blood supply with less blood collections, better smoothed mobile collection activities, better match of blood donations and demand, and low blood inventory. 2) Allowing variable collection frequencies significantly increases the complexity of annual planning and the CPU time needed to solve the model. 3) The optimal weekly bloodmobile collection planning allows not only reducing the total workload of staff personnel but also smoothing the workload over the week. 4) The use of bloodmobile collection configurations allows the weekly planning to be solved to optimality in reasonable time.

Both tactical and operational models can be implemented by our partner EFS and used in daily operations of blood collection.

This work can be extended in several directions. Although annual planning with variable collection frequencies brings significant improvement, its utilization can eventually be more hazardous if the annual demand and parameters needed donation forecast are not well known. Stochastic models or robust optimization models of annual bloodmobile collection planning are interesting alternatives to better take into account uncertain demand and uncertain donations. Another interesting perspective would be the implementation of a discretesimulation model in order to assess the robustness of our analytical models versus in realistic working conditions. Various random parameters may be integrated in the model, such as equipment unavailability, weather conditions, and collection results during the first weeks of the year in terms of donor frequentation.

\section{ACKNOWLEDGMENT}

The authors are grateful to Prof. Olivier Garraud, Dr. Catherine Argaud, Dr. Francoise Boussoulade and the EFS Auvergne-Loire staff for their help in field studies.

\section{REFERENCES}

[1] World Health Organization. WHO Towards 100\% voluntary blood donation: a global framework for action Geneva: World Health Organization, 2010.

[2] EFS. Rapport d'activités Etablissement Français du Sang, 2011. Available http://www.dondusang.net/ in EFS/publications.

[3] J. Beliën, and H. Forcé, "Supply chain management of blood products: A literature review," European Journal of Operational Research, 217(1): 1-16, 2012.

[4] S.O. Crawford, N.G. Reich, M.W. An, R. Brookmeyer, T.A. Louis, K.E. Nelson, E.P. Notari, J. Trouern-Trend, and S. Zou, "Regional and temporal variation in American Red Cross blood donations, 1995 to 2005," Transfusion, 48(8): 1576 -1583, 2008

[5] G. Sahin, H. Süral, and S. Meral, "Locational analysis for regionalization of Turkish Red Crescent blood services," Computers \& Operations Research, 34(3): 692 - 704, 2007.

[6] W.P. Pierskalla, "Supply chain management of blood banks," in Operations research and health care, M.L. Brandeau, F. Sainfort, W.P. Pierskalla. (eds), Springer, 2005.

[7] P. Hervé, "Transfusion medicine in the 2000s, on a reform," Bull Acad Natl Med., 186(2): 379-89, discussion 389-92, 2002.

[8] B. Danic, and A. Beauplet, "La collecte de sang en France : organisation et difficultés," Hématologie, 9(3): 231 - 240, 2003.

[9] R. Courbil, and J.F. Quaranta, Connaître et gérer le risque transfusionnel, Éditions Weka, 2007.

[10] W.N. Rose, P.J. Dayton, and T.J. Raife, "An analysis of mobile blood collection labor efficiency," Transfusion, 51(7): 1609-1612, 2011.

[11] E. Alfonso, V. Augusto, and X. Xie, "Tactical planning of bloodmobile collection systems", Proc. IEEE Conf. Automation Science \& Engineering (CASE’13), Wisconsin-Madison, USA, 2013.

[12] A. Nagurney, A.H. Masoumi, and M. Yu, "Supply chain network operations management of a blood banking system with cost and risk minimization," Comput Manag Sci, 9(2):205-31, 2011. 


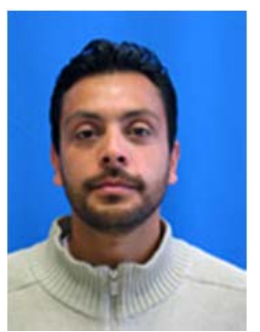

Edgar Alfonso received the Ph.D. degree in industrial engineering from the École Nationale Superieure des Mines de Saint-Étienne (ENSM.SE), Saint-Étienne, France, in 2013.

Currently, he is an Assistant Professor with the Logistics Systems Group, Universidad de la Sabana, Colombia. His research interests include modeling, simulation, optimization of health care systems and their supply chains.

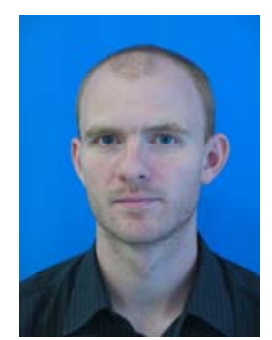

Vincent Augusto received the Ph.D. degree in industrial engineering from the École Nationale Superieure des Mines de Saint-Étienne (ENSM.SE), Saint-Étienne, France, in 2008.

He was a Visiting Scholar with the Centre Interuniversitaire de Recherche sur les Réseaux d'Entreprise, la Logistique et le Transport (CIRRELT), University of Laval, Laval,QC, Canada, in 2009. Currently, he is an Assistant Professor with the Department of Health Care Engineering, ENSM.SE. His research interests include modeling, simulation, optimization of health care systems and their supply chains.

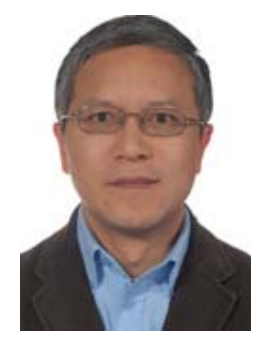

Xiaolan Xie (M'93-SM'10) received his Ph.D degree from the University of Nancy I, Nancy, France, in 1989, and the Habilitation à Diriger des Recherches degree from the University of Metz, France, in 1995.

Currently, he is a Distinguished Professor of industrial engineering, the Head of the Department of Healthcare Engineering of the Center for Biomedical and Healthcare Engineering and the Head of IEOR team of CNRS UMR 6158 LIMOS, Ecole Nationale Supérieure des Mines (ENSMSE), Saint Etienne, France. He is also a Chair Professor and Director of the Center for healthcare engineering at the Shanghai Jiao Tong University, China. Before Joining ENSMSE, he was a Research Director at the Institut National de Recherche en Informatique et en Automatique (INRIA) from 2002 to 2005, a Full Professor at Ecole Nationale d'Ingénieurs de Metz from 1999 to 2002, and a Senior Research Scientist at INRIA from 1990 to 1999. His research interests include design, planning and scheduling, supply chain optimization, and performance evaluation, of healthcare and manufacturing systems. He is author/coauthor of over 250 publications including over 90 journal articles and six books. He has rich industrial application experiences with European industries. He is PI for various national and international projects including ANR-TECSAN HOST on management of winter epidemics, NSF China key project on planning and optimization of health care resources, French Labex IMOBS3 project on home health cares, FP6-IST6 IWARD on swarm robots for health services, FP6-NoE I*PROMS on intelligent machines and production systems, the FP5-GROWTH-ONE project for the strategic design of supply chain networks, the FP5- GRWOTH thematic network TNEE on extended enterprises.

Dr. Xie has been an associate editor for International Journal for Production Research, IEEE Transactions on Automation Science \& Engineering, IEEE Transaction on Automatic Control, IEEE Transactions on Robotics \& Automation and has been on the (conference) editorial board of the IEEE Robotics and Automation Society, Health Systems, and Int. J. Simulation \& Process Modelling. He has a Guest Editor of various special issues on healthcare engineering and manufacturing systems. He is general chair of ORAHS'2007, IPC chair of the IEEE Workshop on Health Care Management WHCM'2010 and IPC member for many other conferences. He is the corresponding co-chair of the IEEE RAS Technical Committee on Automation in Healthcare Management. 